\title{
Temperature-Dependent Sex Determination in Hd-rR Medaka Oryzias latipes: Gender Sensitivity, Thermal Threshold, Critical Period, and DMRT1 Expression Profile
}

\author{
R.S. Hattoria ${ }^{a}$ R.J. Gould ${ }^{\text {a }}$ T. Fujioka ${ }^{a}$ T. Saito ${ }^{b} \quad$ J. Kurita ${ }^{c}$ C.A. Strüssmann ${ }^{a}$ \\ M. Yokota ${ }^{a}$ S. Watanabe ${ }^{a}$ \\ a Department of Marine Biosciences, Faculty of Marine Science, Tokyo University of Marine Science and \\ Technology, Tokyo, b Kumamoto Prefectural Fisheries Research Center, Kumamoto, ' Inland Station, \\ National Research Institute of Aquaculture, Fisheries Research Agency, Mie, Japan
}

\section{Key Words}

DMRT1 - Fertility $\cdot$ High temperature $\cdot$ Medaka $\cdot$

Sex differentiation $\cdot$ Temperature-dependent sex determination

\begin{abstract}
The developmental time and thermal threshold for temperature-dependent sex determination (TSD), gender differences in temperature sensitivity, the fertility of thermally sex reversed fish, and the effect of temperature on the expression of two major sex determination/differentiation genes (DMY/DMRT1bY and DMRT1) were examined in the Hd-rR strain of medaka, Oryzias latipes. Fertilized eggs were exposed from either shortly after fertilization (8-16 cells; embryonic stages 5-6) or from middle embryogenesis (heart development stage; stage 36 ) until hatching to temperatures ranging from $17^{\circ} \mathrm{C}$ to $34^{\circ} \mathrm{C}$. Secondary sexual characteristics, gonadal histology, progeny testing, sex-linked body coloration and gene expression were used to determine phenotypic and genotypic sex. Sex determination was unaffected by low or high temperatures in genotypic (XY) males. In contrast, genotypic (XX) females treated from stag-
\end{abstract}

es 5-6 showed increasing rates of sex reversal into phenotypic males at temperatures above $27^{\circ} \mathrm{C}$ up to $100 \%$ at $34^{\circ} \mathrm{C}$. Thermal manipulation of sex was ineffective after stage 36 , indicating that gonadal fate in medaka is determined considerably earlier than histological differentiation (stage 39). High temperature induced DMRT1 expression in genotypic females, which was observed already from stage 36 . Sex-reversed males had histologically normal testes, were capable of sexual courtship and, with the exception of fish from $34^{\circ} \mathrm{C}$, sired viable progeny when mating with fertile females. These results clarify the pattern of TSD in medaka and provide important clues to understand the mechanism of sex determination in this species. They also suggest that a brief exposure to high temperature early in life could impair the fertility of medaka as adults.

Copyright $\odot 2007$ S. Karger AG, Basel

This project was supported by grants-in-aid from the Ministry of Education, Culture, Sports, Science and Technology of Japan (\#12460085 and \#15201003) and from the Tokyo University of Marine Science and Technology to C.A.S.

\section{KARGER}

Fax +4161306 1234

E-Mail karger@karger.ch

www.karger.com (c) 2007 S. Karger AG, Basel

$1661-5425 / 07 / 0012-0138 \$ 23.50 / 0$

Accessible online at:

www.karger.com/sxd
Dr. Carlos Augusto Strüssmann

Department of Marine Biosciences, Faculty of Marine Science

Tokyo University of Marine Science and Technology

Konan 4-5-7, Minato, Tokyo 108-8477 (Japan)

Tel./Fax +81 35463 0541, E-Mail carlos@s.kaiyodai.ac.jp 
A growing body of experimental evidence suggests that the process of sex determination (or differentiation) in fish is quite sensitive to the effects of environmental temperature [Baroiller et al., 1999; Devlin and Nagahama, 2002; Strüssmann and Nakamura, 2002]. In temperature-dependent (or thermolabile) sex determination (TSD), the thermal conditions during a critical phase in early life can induce the formation of functional ovaries or testes regardless of putative genetic orientation. This phenomenon occurs over a broad range of environmentally relevant temperatures and appears to be not restricted to species from any phylogenetic group, lifestyle or habitat, although it is common and very marked among Atheriniformes [Strüssmann and Patiño, 1995; Strüssmann and Nakamura, 2002]. In this taxon, TSD is indeed the main determinant of the sex ratios in natural populations [Conover and Kynard, 1981; Middaugh and Hemmer, 1987; Strüssmann et al., 2003] and supposedly confers an adaptive advantage to some species (e.g. the Atlantic silverside, Menidia menidia) [Conover, 1984]. However, reports of TSD are available also on dozens of unrelated species, including many which have previously been assumed to have strictly genotypic sex determination (GSD) such as sockeye salmon, Oncorhynchus nerka [Craig et al., 1996], tilapia, Oreochromis niloticus [Baroiller et al., 1995], zebrafish, Danio rerio [Uchida et al., 2004], channel catfish, Ictalurus punctatus [Patiño et al., 1996], and even viviparous species such as Poeciliopsis lucida [Schultz, 1993]. In this context, there appears to be room for the argument that some degree of TSD could very well be the rule rather than the exception among fishes, and that modulation of sex differentiation by temperature probably owes as much to the poor state of development of the mechanisms of sex determination in fish as to poikilothermy itself [Strüssmann and Patiño, 1999]. Moreover, in view of the projected trend of rising average global temperatures and ensuing thermal anomalies, the possibility of effects of temperature on the process of sex differentiation becomes a grave concern to the reproductive health of fish populations.

In medaka Oryzias latipes, the first species of fish in which a main sex determining gene was documented (DMY or DMRT1bY as identified by Matsuda et al. [2002] and Nanda et al. [2002], respectively), sex is generally considered to be strictly determined by the animal's genotype. In this case it is an XX/XY system, as demonstrated by detailed progeny analysis [Aida, 1921; Yamamoto, 1969], cytogenetic analysis [Matsuda et al., 1998], and linkage mapping [Matsuda et al., 2002; Nanda et al., 2002]. Although this sex determining system appears to be fair-

Temperature Effects on Medaka Sex Differentiation ly conserved within the genus Oryzias, only a few species appear to possess the DMY/DMRT1bY gene [reviewed by Schartl, 2004]. Moreover, naturally occurring XX males have been found in several wild [Shinomiya et al., 2004] and laboratory [Nanda et al., 2003] populations of medaka, suggesting that there might be alternate systems of sex determination in this species. Of interest is the observation of Aida [1936] who reported increased proportions of males during hot summers, but little has been done to explain this phenomenon. More recently, studies conducted in our laboratory provided preliminary evidence of TSD in medaka in the form of synergism and antagonism between exposure to low and high temperatures, respectively, and a feminizing hormone (estradiol) during the early stages of sex differentiation [Minamitani and Strüssmann, 2003]. We have since conducted a thorough examination of the effects of temperature on the sex determination of this species. While this paper was in preparation, a report of a similar study on the effects of temperature on sex determination of the Hd-rR and HNI strains of medaka appeared [Sato et al., 2005]. That study provided the first clear evidence of TSD in medaka, showing that embryos of genotypic (XX) females of both strains developed as functional males when exposed to a temperature of $32^{\circ} \mathrm{C}$ between fertilization and hatching. However, no information was provided on the timing of TSD in relation to sex differentiation and on the effects of other temperatures in this process. Likewise, the molecular mechanism of TSD in this species has not been clarified.

In this study, we present the results of experiments spanning the full range of viable temperatures of embryos $\left(15^{\circ} \mathrm{C}\right.$ to $\left.34^{\circ} \mathrm{C}\right)$ on the sex differentiation of both genotypic males and females of the Hd-rR strain of medaka. We also examined the timing of sex determination by thermal treatment of medaka embryos during two different developmental stages and examined the fertility of sex reversed individuals. Finally, we examined the pattern of expression of the putative sex-determining gene $D M Y / D M R T 1 b Y$ and of the autosomal gene DMRT1, which in medaka is associated with spermatogonial differentiation [Kobayashi et al., 2004], in order to obtain clues on the molecular mechanism of high temperatureinduced masculinization in medaka.

\section{Materials and Methods}

Source of Animals

Medaka from the highly inbred Hd-rR strain were a kind gift from the Animal Supply Facilities, National Institute of Radiological Sciences, Chiba, Japan. To obtain fertilized eggs, we set up 
Table 1. Primers used in the analysis of genotypic sex

\begin{tabular}{lll}
\hline Primer & Sequence & Reference \\
\hline PG17.5 & CCGGGTGCCCAAGTGCTCCCGCTG & Matsuda et al. \\
PG17.6 & GATCGTCCCTCCACAGAGAAGAGA & {$[2002]$} \\
EX3.1 & GCAACAGAGAGTTGGATTTACGTCTCA & Shinomiya et al. \\
EX3.2 & CTTTTGACTTCAGTTTGACACATCAATG & {$[2004]$} \\
EX6.1 & GTCATTAACACAACGCACAACAACTT & Shinomiya et al. \\
EX6.2 & AAAAACCAGAAGACCCGAGAGGAAG & {$[2004]$} \\
DMT1K & CAACTTTGTCCAAACTCTGA & Nanda et al. \\
DMT11 & AACTAATTCATCCCCATTCC & {$[2003]$} \\
obB-actin 4F & CTCTGGTCGTACCACTGGTATCG & Present study \\
obB-actin 4R & GCAGAGCGTAGCCTTCATAGATG & \\
\hline
\end{tabular}

nine 20-liter plastic tanks containing 4 adult females and 2 males each. This brood stock was reared in brackish water $(0.1-0.4 \%$ $\mathrm{NaCl})$ at a temperature of $25^{\circ} \mathrm{C}$ and a photoperiod of $14 \mathrm{~L}-10 \mathrm{D}$. Fish were fed Tetramin $\left(\right.$ Tetra $\left.^{\circledR}\right)$ flakes and supplemented with live Artemia nauplii ad libitum.

\section{Embryo Collection and Incubation}

Fertilized eggs were collected daily within $2 \mathrm{~h}$ of spawning, and stocked in Petri dishes with brackish $(0.1-0.4 \% \mathrm{NaCl})$ water and methylene blue (50 ppm) as a prophylactic at a stocking density of up to 50 eggs per dish. To assess the effects of temperature and the thermolabile period, groups of eggs were transferred within $1 \mathrm{~h}$ of collection ( $3 \mathrm{~h}$ from spawning, developmental stages 5-6) [Iwamatsu, 1994] to incubators (IUCHI Mini-Incubator UI-50) pre-set at $17,21,25,27,30,32$ and $34 \pm 0.3^{\circ} \mathrm{C}$ or after a preliminary incubation period of 6 days at $25^{\circ} \mathrm{C}$ (until the heart development stage, or stage 36 ) to incubators set at $15,18,22,25$, $26,30,31$, and $34 \pm 0.3^{\circ} \mathrm{C}$. These experimental temperatures were chosen based on empirical results on the thermal tolerance and hatchability of Hd-rR strain medaka eggs and those of Yamamoto [1975]. After $24 \mathrm{~h}$, unviable eggs were discarded, the chorionic filaments were trimmed off, and the rearing water was exchanged completely for new water previously adjusted to the same temperature. Thereafter, unviable eggs were removed and the water was exchanged every $48 \mathrm{~h}$ until hatching.

\section{Sexing of Fish}

Hatched larvae were immediately separated into groups according to their incubation temperature and reared under the same standard conditions as for brood stock until reaching sexual maturity at about 2-3 months. No attempt was made to discriminate between larvae from different spawnings or parents within the same temperature incubation group because of the high degree of inbreeding of the brood stock. After attaining sexual maturity, each fish was analyzed to determine its phenotypic and genotypic sex. Phenotypic sex was determined by observation of secondary sexual characteristics in fin morphology and anal papilla under a stereomicroscope following identification criteria of Yamamoto [1975] and Iwamatsu [1999]. Phenotypic sex was also examined by light histological analysis of the gonads in selected individuals to confirm the findings of the observation of secondary sex characteristics. For this purpose, fish were fixed in
Bouin's solution for $24 \mathrm{~h}$ and preserved in $70 \%$ ethanol. The trunk region containing the gonads was then processed for histological analysis by routine histological methods. Sagittal histological sections of $6 \mu \mathrm{m}$ of thickness were stained with hematoxylin-eosin and examined under a microscope. The genetic sex of each individual was identified by two methods. First, we observed each fish under the stereomicroscope concomitant with the determination of secondary sexual characteristics and scored the genetic sex based on body pigmentation. This is possible in the Hd-rR strain of medaka because inheritance of sex and body pigmentation are linked: genotypic (XX) females are white and genotypic (XY) males are orange-red [Yamamoto, 1975].

Second, animals were subjected to DNA analysis for the presence of the DMY/DMRT1bY gene. For this purpose, we collected a piece of the caudal fin of all individuals sacrificed for histology and extracted their total DNA using the DNeasy ${ }^{\circledR}$ Tissue Kit (QIAGEN). A PCR analysis was then performed using 4 sets of primers (table 1 ) to cover the DMY gene in its entirety. $\beta$-Actin was amplified as a positive control in the case of primers EX3.1/3.2, EX6.1/6.2, and DMT1K/DMT1l, since genetic females do not show any band with them. The PCR products were analyzed on a $0.9 \%$ agarose gel.

\section{Progeny Test}

For the progeny and fertility test, selected sex-reversed males (genotypic females; see below) from the 32 and $34^{\circ} \mathrm{C}$ groups were mated individually to control brood stock females that were reared throughout at $25^{\circ} \mathrm{C}$ and which had proven fertility records. Eggs were collected daily and checked for fertilization and development. All other rearing procedures for the progeny test were the same as those described above.

\section{Gene Expression Analysis}

Offspring from the cross of control (XX) females with control $(\mathrm{XY})$ and sex-reversed (XX) males from the $32^{\circ} \mathrm{C}$ group was incubated from fertilization to hatching at $25^{\circ} \mathrm{C}$ and $32^{\circ} \mathrm{C}$, respectively. Twelve embryos were sampled from each group at developmental stages 36 and 39 (hatching) and individually analyzed for $D M Y / D M R T 1 b Y$ and DMRT1 gene expression by reverse transcription-PCR analysis following the primers and methods of Nanda et al. [2002]. 
Table 2. Frequencies of sex phenotypes per incubation temperature, timing (embryonic stage) of treatment, and genotypic sex in Hd-rR strain medaka

\begin{tabular}{llllll}
\hline $\begin{array}{l}\text { Incubation } \\
\text { temperature } \\
\left({ }^{\circ} \mathrm{C}\right)\end{array}$ & Genotypic XX & & & Genotypic XY & \\
\cline { 2 - 2 } & Sex phenotype & Sex reversal & & Sex phenotype & Sex reversal \\
\cline { 2 - 3 } & Female Male & & & Female Male & \\
\hline
\end{tabular}

$\begin{array}{lrrrrrl}\text { Incubation from stage } & 5-6(8-16 \text { cells }) & & \\ 17 & 12 & 0 & 0 & 0 & 8 & 0 \\ 21 & 29 & 0 & 0 & 0 & 36 & 0 \\ 25 & 34 & 0 & 0 & 0 & 37 & 0 \\ 27^{\mathrm{a}} & - & 2 & - & - & - & - \\ 30 & 30 & 13 & 30.2 & 0 & 21 & 0 \\ 32 & 9 & 6 & 40.0 & 0 & 12 & 0 \\ 34 & 0 & 13 & 100.0 & 0 & 6 & 0\end{array}$

Incubation from stage 36 (heart development stage)

$\begin{array}{rrrllrl}15 & 24 & 0 & 0 & 0 & 16 & 0 \\ 18 & 5 & 0 & 0 & 0 & 8 & 0 \\ 22 & 21 & 0 & 0 & 0 & 19 & 0 \\ 25 & 32 & 0 & 0 & 0 & 16 & 0 \\ 26 & 36 & 0 & 0 & 0 & 31 & 0 \\ 30 & 16 & 0 & 0 & 0 & 17 & 0 \\ 31 & 37 & 0 & 0 & 0 & 17 & 0 \\ 34 & 35 & 0 & 0 & 0 & 37 & 0\end{array}$

a All but two fish were accidentally lost.

\section{Results and Discussion}

Eggs that were incubated at 17 and $34^{\circ} \mathrm{C}$ from immediately after fertilization hatched in approximately 25 and 7 days, respectively. These results match the developmental times for these temperatures and the thermal tolerance of medaka embryos reported by Yamamoto [1975]. However, we noted that embryos incubated at high temperatures, particularly at $34^{\circ} \mathrm{C}$, frequently showed abnormal embryonic development. The most common types of abnormalities were those in the nervous system such as deformed and atrophied brains, small or missing eyes, and general cranial deformities, and these developmental problems likely led to the reduced viability observed in these groups. Nevertheless, embryos with milder degrees of deformities were in general able to hatch, start exogenous feeding, and grow until adulthood, and these were the animals that were sexed and submitted to progeny testing as described below.

Determination of genotypic and phenotypic sex in sexually mature individuals from all groups was first performed non-destructively by examination of body color and secondary sexual characters, respectively. The results of these analyses were then confirmed by gene amplification and histology, respectively. Overall, the results of sex ratios compiled in table 2 clearly indicate that temperature treatment caused sex reversal from genotypic female into phenotypic male at the highest temperatures $\left(30,32\right.$ and $\left.34^{\circ} \mathrm{C}\right)$. We also observed two sex reversed males at the temperature of $27^{\circ} \mathrm{C}$ but, since most fish in this group were accidentally lost, the actual proportion of sex reversal in this group could not be calculated. Determination of genotypic sex from body color was very accurate as all XX individuals, including sex reversed males and normal females, showed only the autosomal $D M R T 1$ fragment by amplification with primers PG17.5 and 17.6 and none with the other three primer sets (fig. 1). The XX genetic makeup of sex reversed males was also confirmed in the progeny testing, as they sired all female (XX) progeny when mated to control females (results not shown). In genotypic males, however, primers PG17.5 and 17.6 amplified two fragments, a larger one $(\sim 1 \mathrm{~kb})$ corresponding to DMRT1 and a smaller one $(\sim 0.7 \mathrm{~kb})$ corresponding to $D M Y / D M R T 1 b Y$. In these animals, a single band corresponding to DMYIDMRT1bY was also amplified with the other three primer sets (fig. 1).

The proportion of sex-reversal of genotypic females at the temperature of $32^{\circ} \mathrm{C}$ obtained in this study $(40 \%)$ was grossly equivalent to that reported by Sato et al. [2005] for the same temperature (24\%) if slight differences in tem- 


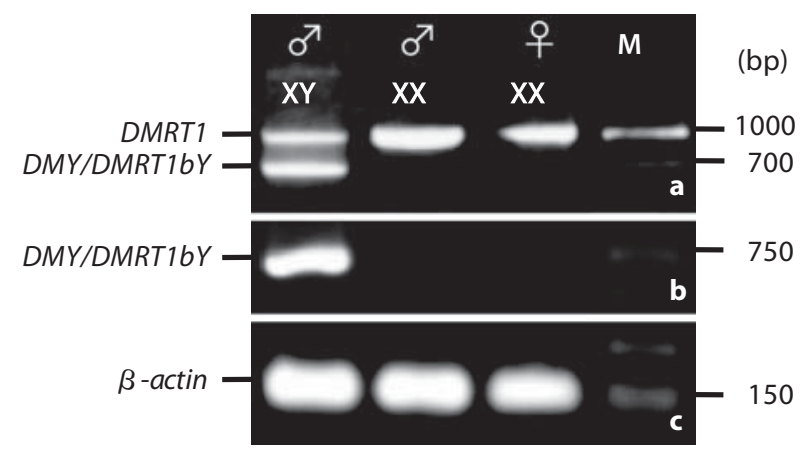

Fig. 1. Genetic screening of normal and sex reversed medaka by amplification of DMY/DMRT1bY using the primers P17.5 and P17.6 (a) and E3.1 and 3.2 (b). Note that for the first set of primers the autosomal DMRT1 is also amplified. The positive control for primers E3.1 and 3.2 ( $\beta$-actin) is shown in c. The other two primer sets produced the same pattern as found in $\mathbf{b} . \mathrm{M}=$ Molecular weight marker.

perature between the two experiments are taken into consideration. This study, however, shows for the first time that the efficiency of sex reversal by temperature in genotypic females of Hd-rR medaka increases proportionally with temperature above $27^{\circ} \mathrm{C}$, reaching $100 \%$ at $34^{\circ} \mathrm{C}$ (table 2). On the contrary, our results suggest that sex differentiation in genotypic (XY) males is greatly resilient to thermal effects, as sex-reversed females were not found in any of the temperature groups. This was rather surprising, as we expected to find also sex reversal at the low temperatures. Our expectation was based on studies showing that the expression of various genes purportedly involved in the process of sex differentiation in fish is affected by environmental temperature in a similar way as by treatment with exogenous steroid hormone treatments [reviewed in Devlin and Nagahama, 2002; Strüssmann and Nakamura, 2002]. Moreover, in support of our assumption, Minamitani and Strüssmann [2003] showed that the efficiency of feminization by administration of estradiol to Hd-rR medaka embryos increased by incubating fertilized eggs at low temperature during hormone treatment. The results of this study, however, suggest that low temperature alone is not sufficient to corrupt the putative genetic mechanism of sex differentiation in medaka although we cannot rule out the possibility that exposure to even lower temperatures (for short duration to guarantee viability) could still cause sex reversal.
The above results have important implications for the discussion of the mechanism and evolution of sex determination in medaka. For example, one puzzling aspect of sex determination in this species is the paradoxical facets of the DMY/DMRT1bY gene: it is both new and old (= disappearing), and it is both sufficient but not absolutely necessary for testicular differentiation [see Schartl, 2004]. In fact, two studies [Nanda et al., 2003; Schartl, 2004] suggest that autosomal modifiers could be implicated in male fate determination in medaka. The findings of this study and those of Sato et al. [2005] add a new dimension to this discussion, as they show that part, if not all, of the masculinizing role of DMY/DMRT1bY could readily be taken over by (high) environmental temperature. Thus, whereas Schartl [2004] has envisioned the loss of the Y chromosome of medaka and its replacement by other genetic factors, we suggest that environmental temperature may actually accelerate its demise. Indeed, it is noteworthy that sex reversal of genotypic females was observed at a temperature of $27^{\circ} \mathrm{C}$ in the HNI [Sato et al., 2005] and Hd-rR (this study) strains, so it is possible that at least part of the F1 and F2 XX males attributed by Nanda et al. [2003] to autosomal modifiers could have been sex reversed by the rearing temperature in their experiments $\left(27^{\circ} \mathrm{C}\right)$. Given the occurrence of sex reversal at environmentally realistic temperatures $\left(27-32^{\circ} \mathrm{C}\right)$ and projected trends in global warming, the postulated disappearance of the Y chromosome could be even accelerated by the 'dilution effect', in which sex reversed XX males compete with $\mathrm{XY}$ males for mating with $\mathrm{XX}$ females, leading to a gradual reduction of the proportion of XY individuals in the population [assuming the persistence of the masculinizing temperatures; see Kanaiwa and Harada, 2002]. Thus, our results point to a new paradox concerning the Y chromosome of medaka: that it is both strong, as it is able to fend off the feminizing effects of (low) temperature, but at the same time weak, as it can be readily replaced by alternative genetic and environmental factors and unable to prevent its own extinction.

A second important finding of this study was that the thermally sensitive period of sex differentiation in medaka embryos lies between developmental stages 5-6 (8 to 16 cells) and 36 (heart development stage), as thermal treatments only effectively induced sex reversal in this time/developmental window. This result conflicts with the prevailing view that gonadal sex is determined in medaka around the later half of embryogenesis (between stages 36 and 39), as implied from the results of histological analysis of gonadal sex differentiation [particularly germ cell proliferation; Satoh and Egami, 1972; 
Quirk and Hamilton, 1973; Yoshikawa and Oguri, 1981; Kobayashi et al., 2004]. It also contradicts estimates obtained from hormonal manipulation of sex in this species; for such treatments are $100 \%$ effective even when started after the heart development (or eyed-egg) stage [Iwamatsu, 1999; Minamitani and Strüssmann, 2003], a fact commonly taken as an indication that gonadal sex is still not determined at that time. It is worth mentioning, however, that sex steroid hormones are potent inducers of sex that can override putative processes [even in the adult gonads; Bhandari et al., 2006]. On the contrary, wherever it has been possible to determine the time of sex determination by other means, such as thermal manipulation of sex in TSD species, for example, it was shown that sex is determined considerably earlier than the first signs of gonadal sex differentiation [Conover and Fleisher, 1986; Baroiller et al., 1995; Strüssmann et al., 1997; Wang and Tsai, 2000]. For the above reasons, we consider that our results obtained by thermal manipulation reflect more closely the time of natural sex determination in (untreated) medaka than previous determinations using either histology or hormonal manipulation.

This revised estimate of the period of natural sex determination in medaka may be fundamental for the functional analysis of the role of genetic factors in this process. The DMY/DMRT1bY gene, for instance, is considered as the prime testis-determining factor in this species, with functional (but not necessarily structural) analogy to the sex determining genes in other taxa [Matsuda et al., 2002; Nanda et al., 2002]. Kobayashi et al. [2004] observed expression at stage 37, when the primordial germ cells (PGCs) have already migrated into the gonadal anlagen and before the appearance of a sexual dimorphism in the number of germ cells at stage 39. Expression of DMY/ $D M R T 1 b Y$ was detected specifically in Sertoli-like cells surrounding PGCs, leading the authors to suggest that it regulates the differentiation of these cells and/or the proliferation/differentiation of germ cells and thereby induces testicular differentiation. Nanda et al. [2002], on the other hand, detected DMY/DMRT1bY expression already from the neurula stage (stages 17-18), but did not elaborate on a role at this early stage. Thus, although $D M Y /$ $D M R T 1 b Y$ is presumably located far upstream in the male pathway, its target genes still remain unknown [Nanda et al., 2002; Schartl, 2004]. It is notable that the early window of expression observed by Nanda et al. [2002] coincides temporally with the thermally sensitive period of masculinization determined in this study, and that both occur even before migration of PGCs and their entry into the germinal ridges. These facts suggest a similar key role

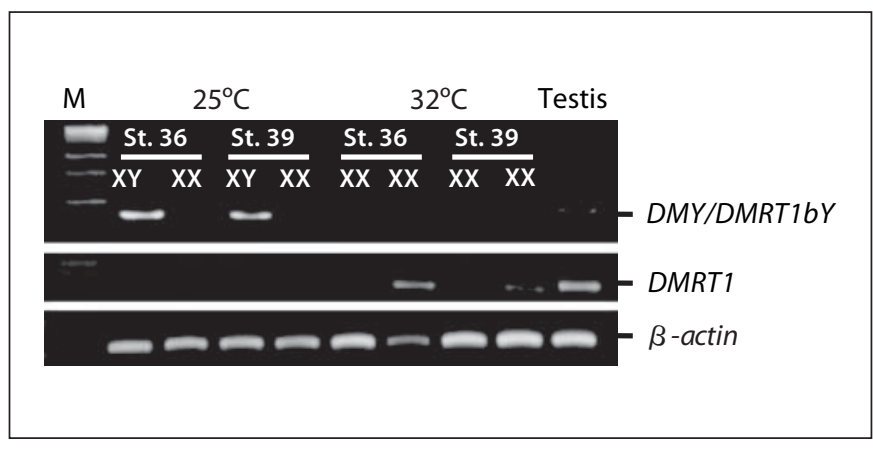

Fig. 2. Reverse transcription-PCR analysis of $D M Y / D M R T 1 b Y$ and DMRT1 gene expression in XX and XY medaka embryos of two developmental stages (36 and 39$)$ at sexually neutral $\left(25^{\circ} \mathrm{C}\right)$ and masculinizing $\left(32^{\circ} \mathrm{C}\right)$ temperatures. For clarity, only representative results for each genotype, incubation temperature, developmental stage, and pattern of DMRT1 gene expression are shown from among the twelve embryos available for each condition. Results for adult medaka testis are included for comparison. $\mathrm{M}=$ Molecular weight marker.

for these two factors during early development, although they do not prove that the mechanism of action in both cases is the same, as for example water temperature could have cumulative effects on other genes. As expected in the absence of the gene [see also Nanda et al., 2002; Kobayashi et al., 2004], expression of DMY/DMRT1bY was not observed in any of the genotypic female (XX) embryos regardless of developmental stage and incubation temperature (fig. 2). In spite of the absence of DMY/ $D M R T 1 b Y$, however, substantial amounts of DMRT1 mRNA were found in 6 out of $12 \mathrm{XX}$ embryos incubated at $32^{\circ} \mathrm{C}$, a fact not observed at the 'sexually neutral' temperature of $25^{\circ} \mathrm{C}$. This finding is in good agreement with the proportion of sex-reversed males found at this temperature (e.g. 40\%; table 2). This is the first demonstration that genotypic female medaka embryos express this crucial gene for testis formation in response to masculinizing temperatures. Likewise, $D M R T 1$ expression at $32^{\circ} \mathrm{C}$ was observed as early as developmental stage 36 . This contrasts with a temperature of $25^{\circ} \mathrm{C}$, where it is observed only after hatching [stage 39; this study; Nanda et al., 2002; Kobayashi et al., 2004]. Thus, high temperature greatly accelerates the cascade of molecular events leading to testicular differentiation. Further studies must examine how high temperature triggers the expression of $D M R T 1$, if this early expression actually induces premature testicular differentiation, and further clarify the molecular cascade leading to gonadal sex differentiation in medaka. 
Fig. 3. Sagittal histological sections of gonads from normal female (a, b), normal male $(\mathbf{c}, \mathbf{d})$ and sex-reversed male from the $34^{\circ} \mathrm{C}$ treatment $(\mathbf{e}, \mathbf{f})$. Figures on the left and right were all taken at the same magnification. Figures on the right are magnifications of the respective boxes on the left. Symbols: oc = ovarian cavity; po $=$ perinucleolar oocyte; $o g$ = oogonia; sg = spermatogonia; $s o=$ spermatocytes; $\mathrm{sp}=$ sperm cells.

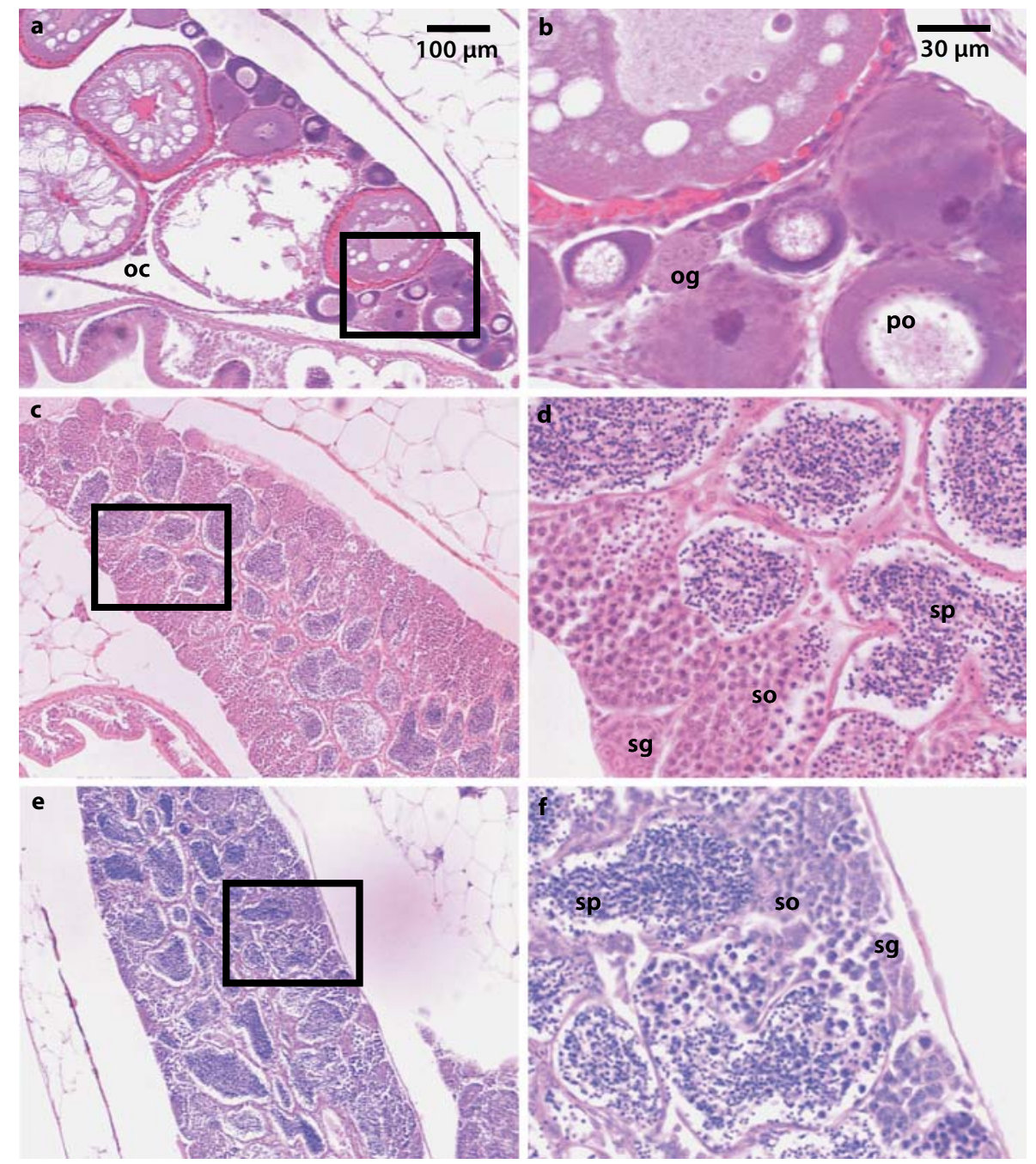

Histological analysis of sex-reversed males from the high temperature groups revealed that their testes were morphologically normal, presenting the characteristic tubular organization and cysts of germ cells (spermatogonia, spermatocytes and spermatozoa) [Kanamori et al., 1985], and were undistinguishable from those of males from the control group $\left(25^{\circ} \mathrm{C}\right.$; fig. 3$)$. We did not find any differences between females from the various groups either and intersex features were never observed. The progeny tests showed that sex-reversed males from the 32 and $34^{\circ} \mathrm{C}$ groups were capable of normal sexual courtship but only animals from the former treatment produced viable offspring. The fertility of animals from $32^{\circ} \mathrm{C}$ agrees with the results of hormonal [Yamamoto, 1958] and temperature treatments by Sato et al. [2005], who showed that XX males are in general functional, although we cannot discard the possibility that they are reproductively inferior to XY males [see Fineman et al., 1975]. The infertility of sex reversed males from the $34^{\circ} \mathrm{C}$ treatment, on the other hand, resembles in all respects the infertile XX males observed by Yamamoto [1958] after hormonal manipulation in that they had normal testicular histology, produced mature spermatozoa, mated actively, but did not sire viable progeny. In the present study, it might represent a problem of orchestration between the neural system and the gonads since fish at the highest temperatures frequently showed cranial and brain abnormalities. This possibility and that of genotoxic effects of heat [Anitha et al., 2000] should be examined in further studies.

The results of this study show unmistakably that the process of gonadal sex differentiation in Hd-rR medaka is under the control of genetic and environmental (temperature) factors at a very early stage of embryonic development. They also reveal that a brief exposure to high 
temperatures early in life could greatly impair the fertility of fish as adults. Thus, medaka constitutes an invaluable research tool to study the effects of temperature on the process of sex differentiation and provides the opportunity to test the strength of a clear sex determination mechanism, which shares a high degree of homology with that of other vertebrates, in a poikilothermic context. Such studies may help to shed light on the mechanism of TSD in fish and to provide tools to forecast the impact of global warming and climatic change on the reproduction of fish populations.

\section{Acknowledgements}

We would like to thank Dr. Y. Ishikawa (Animal Supply Facilities, National Institute of Radiological Sciences, Chiba, Japan) for the kind gift of Hd-rR medaka eggs. We are indebted to N. Minamitani and $\mathrm{Xu}$ Tian Tian for their assistance in the experiments.

\section{References}

Aida T: On the inheritance of color in a freshwater fish Aplocheilus latipes Temminck and Schlegel, with special reference to sex-linked inheritance. Genetics 6:554-573 (1921).

Aida T: Sex reversal in Aplocheilus latipes and a new explanation of sex determination. Genetics 21:136-153 (1936).

Anitha B, Chandra N, Gopinath PM, Durairaj G: Genotoxicity evaluation of heat shock in gold fish (Carassius auratus). Mut Res 469: $1-8$ (2000).

Baroiller J-F, Chourrout D, Fostier A, Jalabert B: Temperature and sex chromosomes govern sex ratios on the mouthbrooding cichlid fish Oreochromis niloticus. J Exp Zool 273:216223 (1995).

Baroiller J-F, Guiguen Y, Fostier A: Endocrine and environmental aspects of sex differentiation in fish. Cell Mol Life Sci 55:910-931 (1999).

Bhandari RK, Nakamura M, Nagahama Y: Evidence for existence of sexual plasticity in the adult gonochoristic fish. Abstracts 4th Intern Symp Biol Vert Sex Det, pp 101-102 (Kona, Hawaii, 2006).

Conover DO: Adaptive significance of temperature-dependent sex determination in a fish. Am Nat 123:297-313 (1984).

Conover DO, Fleisher MH: Temperature-sensitive period of sex determination in the Atlantic silverside, Menidia menidia. Can J Fish Aquat Sci 43:514-520 (1986).

Conover DO, Kynard BE: Environmental sex determination: interaction of temperature and genotype in a fish. Science 213:577-579 (1981).

Craig JK, Foote CJ, Wood CC: Evidence for temperature-dependent sex determination in sockeye salmon (Oncorhynchus nerka). Can J Fish Aquat Sci 53:141-147 (1996).

Devlin RH, Nagahama Y: Sex determination and sex differentiation in fish: an overview of genetic, physiological, and environmental influences. Aquaculture 208:191-364 (2002).
Fineman R, Hamilton J, Chase G: Reproductive performance of male and female phenotypes in three sex chromosomal genotypes (XX, $\mathrm{XY}, \mathrm{YY}$ ) in the killifish, Oryzias latipes. J Exp Zool 192:349-354 (1975).

Iwamatsu T: Stages of normal development in the medaka Oryzias latipes. Zool Sci 11:825839 (1994).

Iwamatsu T: Convenient method for sex reversal in a freshwater teleost, the medaka. J Exp Zool 283:210-214 (1999).

Kanaiwa M, Harada Y: Genetic risk involved in stock enhancement of fish having environmental sex determination. Popul Ecol 44:715 (2002).

Kanamori A, Nagahama Y, Egami N: Development of the tissue architecture in the gonads of the medaka Oryzias latipes. Zool Sci 2: 695-706 (1985).

Kobayashi T, Matsuda M, Kajiura-Kobayashi H, Suzuki A, Saito N, et al: Two DM domain genes, $D M Y$ and $D M R T 1$, involved in testicular differentiation and development in the medaka, Oryzias latipes. Dev Dyn 231:518526 (2004).

Matsuda M, Matsuda C, Hamaguchi S, Sakaizumi M: Identification of sex chromosomes of the medaka, Oryzias latipes, by fluorescence in situ hybridization. Cytogenet Cell Genet 82:257-262 (1998)

Matsuda M, Nagahama Y, Shinomiya A, Sato T, Matsuda C, et al: A Y-specific, DM-domain gene, $D M Y$, is required for male development in the medaka (Oryzias latipes). Nature 417: 559-563 (2002).

Middaugh DP, Hemmer MJ: Influence of environmental temperature on sex-ratios in the tidewater silverside, Menidiapeninsulae (Pisces: Atherinidae). Copeia 1987:958-964 (1987).

Minamitani N, Strüssmann CA: Effect of temperature on the efficiency of feminization of medaka (Oryzias latipes) by hormonal (estradiol) manipulation. Fish Physiol Biochem 28:163-164 (2003).
- Nanda I, Kondo M, Hornung U, Asakawa S, Winkler C, et al: A duplicated copy of $D M R T 1$ in the sex-determining region of the $\mathrm{Y}$ chromosome of the medaka, Oryzias latipes. Proc Natl Acad Sci USA 99:11778-11783 (2002).

Nanda I, Hornung U, Kondo M, Schmid M, Schartl M: Common spontaneous sex-reversed XX males of the medaka Oryzias latipes. Genetics 163:245-251 (2003).

Patiño R, Davis KB, Schoore JE, Uguz C, Strüssmann CA, et al: Sex differentiation of channel catfish gonads: normal development and effects of temperature. J Exp Zool 276:209218 (1996)

Quirk JG, Hamilton JB: Number of germ cells in known male and known female genotypes of vertebrate embryos (Oryzias latipes). Science 180:963-964 (1973).

Sato T, Endo T, Yamahira K, Hamaguchi S, Sakaizumi M: Induction of female-to-male sex reversal by high temperature treatment in medaka, Oryzias latipes. Zool Sci 22: $985-$ 988 (2005).

Satoh N, Egami N: Sex differentiation of germ cells in the teleost, Oryzias latipes, during normal embryonic development. J Embryol Exp Morphol 28:385-395 (1972).

Schartl M: A comparative view on sex determination in medaka. Mech Dev 121:639-645 (2004).

Schultz RJ: Genetic regulation of temperaturemediated sex ratios in the livebearing fish Poeciliopsis lucida. Copeia 1993:1148-1151 (1993).

Shinomiya A, Otake H, Togashi K, Hamaguchi S, Sakaizumi M: Field survey of sex-reversal in the medaka, Oryzias latipes: Genotypic sexing of wild populations. Zool Sci 21:613619 (2004)

Strüssmann CA, Nakamura M: Morphology, endocrinology, and environmental modulation of gonadal sex differentiation in teleost fishes. Fish Physiol Biochem 26:13-29 (2002). 
Strüssmann CA, Patiño R: Temperature manipulation of sex differentiation in fish, in Goetz FW, Thomas P (eds): Proceedings of the Fifth International Symposium on the Reproductive Physiology of Fish (FishSymp95, Austin, Texas 1995).

Strüssmann CA, Patiño R: Sex determination, environmental, in Knobil E, Neill JD (eds): Encyclopedia of Reproduction, Vol. 4, pp 402-409 (Academic Press, San Diego 1999).

Strüssmann CA, Saito T, Usui M, Yamada H, Takashima F: Thermal thresholds and critical period of thermolabile sex determination in two atherinid fishes, Odontesthes bonariensis and Patagonina hatcheri. J Exp Zool 278: 167-177 (1997).
Strüssmann CA, Oikawa T, Otake T, Kasuga S: Potential use of otolith microchemistry for field studies of temperature-dependent sex determination and gonadal degeneration in fish. Fish Physiol Biochem 28:129-130 (2003).

Uchida D, Yamashita M, Kitano T, Iguchi T: An aromatase inhibitor or high water temperature induce oocyte apoptosis and depletion of $\mathrm{P} 450$ aromatase activity in the gonads of genetic female zebrafish during sex-reversal. Comp Biochem Physiol 137A:11-20 (2004).

Wang LH, Tsai CL: Effects of temperature on the deformity and sex differentiation of tilapia, Oreochromis mossambicus. J Exp Zool 286: 534-537 (2000).
Yamamoto T: Artificial induction of functional sex-reversal in genotypic females of the medaka (Oryzias latipes). J Exp Zool 137:227263 (1958).

Yamamoto T: Sex differentiation, in Hoar WS, Randall DJ (eds): Fish Physiology, Vol. III. Reproduction and Growth. Bioluminescence, Pigments, and Poisons (Academic Press, New York 1969).

Yamamoto T: Medaka (Killifish): Biology and Strains (Keigaku, Tokyo 1975).

Yoshikawa H, Oguri M: Ovarian differentiation in the medaka, Oryzias latipes, with special reference to the gradient of the differentiation. Bull Jpn Soc Sci Fish 47:43-50 (1981). 